\title{
A Universal Curve Representing the Concentration and Molecular Weight Dependences of Sedimentation Coefficient
}

\author{
Lech GMACHOWSKI \\ Institute of Physical Chemistry, Polish Academy of Sciences; 01-224 Warsaw, Poland
}

(Received January 16, 1990)

\begin{abstract}
A new model is developed for the solvent flow relative to a swarm of polymer coils in a dilute solution and blobs in a semidilute range. The basic idea is that each coil and blob consist of monosized non-porous spheres and pure solvent. Using the porous [Polym. J., 18, 783 (1986)] and hard sphere models, generalized coordinates are derived to correlate the concentration and molecular weight dependences of sedimentation coefficient for any polymer-solvent system onto a single curve. The analytical expression for the portrayal of the curve is also presented. The theory is verified for sedimentation coefficient measured for both a polymer-solvent system at the theta temperature and a polymer-good solvent system.

KEY WORDS Polymer Solution / Hard Sphere Model / Generalized Sedimentation Coefficient /
\end{abstract}

In a previous paper, ${ }^{1}$ a model was presented for the fluid flow relative to a swarm of identical permeable spheres, and it proved to give a good description ${ }^{1,2}$ of the concentration dependence of the sedimentation coefficient $s$.

$$
s=\left(\rho_{\mathrm{s}}-\rho_{1}\right)(1-\varepsilon) /\left(\mu \lambda^{2}\right)
$$

where $\rho_{\mathrm{s}}$ and $\rho_{1}$ are the solute density and solvent density, respectively, $1-\varepsilon$ is the solute volume fraction in the solution, $\mu$ is the solvent viscosity, and $\lambda$ is the reciprocal square root of solution permeability. The model is valid in both dilute and semidilute regimes and for different polymer-solvent systems. It predicts an interdependence of three dimensionless parameters $P R, \lambda R$, and $\delta^{3}$

$$
f\left(P R, \lambda R, \delta^{3}\right)=0
$$

where $P$ is the reciprocal square root of permeability of either coil or blob, dependent on whether the solution is dilute or semidilute, $R$ is the coil or blob radius, and $\delta^{3}$ is the volume fraction occupied by coils or blobs

$$
\delta^{3}=(1-\varepsilon) /\left(1-\varepsilon_{\text {coil }}\right)
$$

or

$$
\delta^{3}=(1-\varepsilon) /\left(1-\varepsilon_{\mathrm{blob}}\right)
$$

$1-\varepsilon_{\text {coil }}$ and $1-\varepsilon_{\mathrm{blob}}$ being the solute volume fractions in the coil and blob, respectively.

It was also shown that if we know the form of the function describing the permeability coefficient of a given semidilute polymer solution

$$
\lambda=\text { const }_{1}(1-\varepsilon)^{n}
$$

the theoretical lines of $\ln \lambda-\ln (1-\varepsilon)$ may be generated by eq 2 from the dilute-semidilute transition points corresponding to given molecular weights of the polymers dissolved. These lines, which were shown to be fulfilled by the experimental data, may be condensed to a single generalized line being the $\ln \lambda R-$ $\ln \delta^{3}$ dependence. This dependence is given by eq 2 utilizing the constancy of $P R$-value, deduced in. ${ }^{1}$ The procedure to recalculate the experimental sedimentation data into the generalized ones is also available. It was shown that the $P R$-value is a unique function of the slope $n$ of eq 5, so we are able to correlate onto 
a single curve the sedimentation data of not only the system analyzed but all polymer-solvent systems of the same $n$-value measured for solutions at different concentrations, containing polymers of different molecular weights.

Nyström et $a l^{3}$ presented similar master curves describing the concentration and molecular weight dependences of the sedimentation coefficient. The curves have another theoretical basis and good experimental support. Each of the universal curves, however, is valid for a given polymer and any solvent of the same quality (the $n$-value saved).

This paper is devoted to show, both theoretically and experimentally, that the sedimentation data points of any polymersolvent system may be condensed onto a single curve.

\section{THE HARD SPHERE MODEL}

The main idea of the model is illustrated by Figure 1. There is assumed that either the coils in the dilute regime or blobs in semidilute range of polymer concentration consist of monosized non-porous spheres and pure solvent. Increasing the concentration of a semidilute solution, the number of spheres in a blob decreases. At a concentration high enough each blob contains only one sphere, so the solution may be represented by a mixture of uniformly dispersed hard spheres. At this concentration,

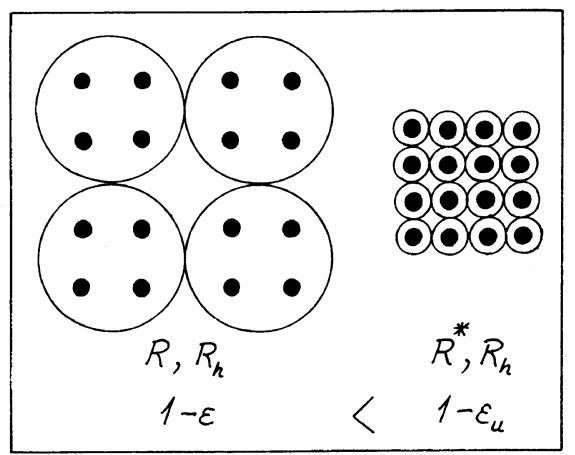

Figure 1. The structure of the hard sphere model. which may be termed as the uniformity concentration, the permeability can be calculated by eq 2 and 4 simplified for $P R \rightarrow \infty$ and $1-\varepsilon_{\mathrm{blob}}=1$ to get

$$
f\left(\lambda R_{\mathrm{h}}, 1-\varepsilon_{\mathrm{u}}\right)=0
$$

where $R_{\mathrm{b}}$ is the hard sphere radius and $1-\varepsilon_{\mathrm{u}}$ is the uniformity solute volume fraction. The permeability at the uniformity concentration may be also evaluated by eq 2 taking into account the real blob porosity and permeability. The blob weight at this concentration is also possible to express in two ways. Hence

$$
R^{*}\left(1-\varepsilon_{\mathrm{blob}}^{*}\right)^{1 / 3}=R_{\mathrm{h}}
$$

where the symbol * relates to the porous blob parameters at the uniformity concentration.

As shown in ${ }^{4}$ the whole range of semidilute solution is equivalent to the inflection point of the line $\ln \lambda R-\ln \delta^{3}$ for a given $P R$-value. The coordinates of the inflection point are marked as follows

$$
\begin{aligned}
P R & =\text { const }_{2} \\
\lambda R & =\text { const }_{3} \\
\delta^{3} & =\text { const }_{4}
\end{aligned}
$$

All of them are functions of $n$-value and may be calculated by eq 2 . From eq 4 and 10 we get

$$
1-\varepsilon_{\mathrm{u}}=\text { const }_{4}\left(1-\varepsilon_{\text {blob }}^{*}\right)
$$

From eq 7 and 11 we obtain

$$
R^{*} / \text { const }_{4}{ }^{1 / 3}=R_{\mathrm{h}} /\left(1-\varepsilon_{\mathrm{u}}\right)^{1 / 3}
$$

Multiplying both sides of eq 12 by $\lambda$ and taking into account eq 9 , we derive

$$
\text { const }_{3} / \text { const }_{4}{ }^{1 / 3}=\lambda R_{\mathrm{h}} /\left(1-\varepsilon_{\mathrm{u}}\right)^{1 / 3}
$$

The left side of eq 13 is a function of $n$, and the right side is possible to calculate by eq 6 . Using eq 5 and 13 we get

$$
\text { const }_{1} R_{\mathrm{h}}=\text { const }_{3} \text { const }_{4}{ }^{-1 / 3}\left(1-\varepsilon_{\mathrm{u}}\right)^{(1-3 n) / 3}
$$

Assuming some $n$-values consecutively, we are able to calculate const ${ }_{3}$ and const 4 by eq 
Table I. The values of the solute volume fraction in the blob at the uniformity concentration for different $n$-values

\begin{tabular}{clllll}
\hline$n$ & 0.7 & 0.8 & 1 & 1.33 & $\infty$ \\
$1-\varepsilon_{\text {blob }}^{*}$ & 0.211 & 0.322 & 0.480 & 0.636 & 1 \\
\hline
\end{tabular}

2 , then $1-\varepsilon_{\mathrm{u}}$ and $\lambda R_{\mathrm{h}}$ by eq 6 and $13,1-\varepsilon_{\mathrm{blob}}^{*}$ by eq 11 , and finally const $R_{1}$ by eq 14 . The calculations were performed for the range of $n$-values from 0.7 to 1.33 which is wider than the theroretical interval between the theta and good solvents being $0.770-1$, according to. ${ }^{3}$ The exponent characterizing the blob weight dependence of the blob radius is equal to $n /(3 n-1)$. Since the exponent must be $1 / 3$ for hard spheres, consequently $n \rightarrow \infty$.

The values of the solute volume fraction in the blob at the uniformity concentration are presented in Table I. Besides, the following correlation was derived from the results obtained

$$
\text { const }_{1} R_{\mathrm{h}} \cong 11.6 n^{1.82}
$$

which permits to evaluate the hard sphere radius from experimental values of const ${ }_{1}$ and $n$.

Assume that the polymer dissolved is of radius $R^{*}$ and solute volume fraction in the coil $1-\varepsilon_{\mathrm{blob}}^{*}$. At the uniformity concentration the equantities

$$
\begin{gathered}
\lambda R\left(1-\varepsilon_{\mathrm{blob}}^{*}\right)^{1 / 3} \\
\delta^{3}\left(1-\varepsilon_{\mathrm{blob}}^{*}\right)
\end{gathered}
$$

are equal to the corresponding values for the hard sphere system $\lambda R_{\mathrm{h}}$ and $1-\varepsilon_{\mathrm{u}}$, respectively, due to eq 7 and 4 . At any lower concentration, the resulting permeability of the two systems should be, at least approximately, the same, to support the hard sphere model. To check this, the values given by the formula 16 were calculated by eq 2 and 3 for consecutive values described by the formula 17 for the $n$-range of $0.7-1.33$, utilizing the values given in Table I. The results are compared in Table II with those computed for hard spheres $(n \rightarrow \infty)$ by putting
Table II. The $\lambda R\left(1-\varepsilon_{\text {blob }}^{*}\right)^{1 / 3}-\delta^{3}\left(1-\varepsilon_{\text {blob }}^{*}\right)$ dependences for different $n$-values

\begin{tabular}{llllll}
\hline & \multicolumn{5}{c}{$n$} \\
$\delta^{3}\left(1-\varepsilon_{\text {blob }}^{*}\right)$ & \multicolumn{7}{c}{0.7} & 0.8 & 1 & 1.33 & $\infty$ \\
\cline { 2 - 6 } & & & & & \\
\hline 0.001 & 0.0758 & 0.0758 & 0.0754 & 0.0748 & 0.0728 \\
0.002 & 0.110 & 0.110 & 0.109 & 0.109 & 0.105 \\
0.004 & 0.161 & 0.161 & 0.160 & 0.159 & 0.154 \\
0.007 & 0.221 & 0.221 & 0.220 & 0.218 & 0.211 \\
0.01 & 0.271 & 0.271 & 0.270 & 0.267 & 0.258 \\
0.02 & 0.411 & 0.412 & 0.410 & 0.406 & 0.390 \\
0.04 & 0.637 & 0.644 & 0.643 & 0.636 & 0.608 \\
0.07 & 0.928 & 0.951 & 0.957 & 0.949 & 0.899 \\
0.1 & 1.19 & 1.24 & 1.27 & 1.26 & 1.18 \\
0.2 & & & 2.35 & 2.42 & 2.25 \\
0.3 & & & & 3.92 & 3.71 \\
\hline
\end{tabular}

$P R \rightarrow \infty, 1-\varepsilon_{\text {coil }}=1$. As we can see, the values given by the formula 16 for consecutive values described by eq 17 are almost the same in the $n$-range of $0.7-1.33$ and very close to the results obtained for hard spheres. So the corresponding theoretical curves drawn in the coordinate system under consideration would be practically indistinguishable. The line valid for hard spheres may be thus treated as the theoretical expression for a universal curve. This is possible to derive from the following equation resulting from eq 2 and 3 by putting $P R \rightarrow \infty$ and $1-\varepsilon_{\text {coil }}=1$.

$$
\frac{(\lambda R)^{2}}{1-\varepsilon}=\frac{\frac{90 a}{1-\varepsilon}(a+b)+9 a(2 a+c)}{5(a+b)^{2}+(2 a+c)(2 a+d)}
$$

where

$$
\begin{aligned}
a= & \frac{\lambda R}{(1-\varepsilon)^{1 / 3}}+1 \\
b= & -\frac{1}{(1-\varepsilon)^{2 / 3}}\left[\frac{\lambda R}{(1-\varepsilon)^{1 / 3}}+3\right] \\
c= & -\frac{2}{(\lambda R)^{2}(1-\varepsilon)}\left[\frac{(\lambda R)^{3}}{1-\varepsilon}+6 \frac{(\lambda R)^{2}}{(1-\varepsilon)^{2 / 3}}\right. \\
& \left.+45 \frac{\lambda R}{(1-\varepsilon)^{1 / 3}}+45\right] \\
d= & -2 \lambda R
\end{aligned}
$$


The quantities 16 and 17 have been recognized as generalized coordinates to correlate the concentration dependence of permeability for dissolved polymers of the molecular weight equal to the blob weight at the uniformity concentration of different $n$-values. The molecular weight may be higher, however, since the dependence $\lambda R-\delta^{3}$ holds for a given $n$-value independently of molecular weight. ${ }^{1}$ So the dependence $\lambda R\left(1-\varepsilon_{\text {blob }}^{*}\right)^{1 / 3}-$ $\delta^{3}\left(1-\varepsilon_{\mathrm{blob}}^{*}\right)$ will also hold since $1-\varepsilon_{\mathrm{blob}}^{*}$ depends only on $n$. As a consequence, the semidilute range will appear as a single point because of the constancy of the generalized coordinates in this region resulting from eq 9 and 10 .

\section{GENERALIZED SEDIMENTATION COEFFICIENT}

To show how the theory agrees with experiment, the general coordinate given by the formula 16 was combined with the second one to get

$$
\begin{aligned}
& {\left[\lambda R\left(1-\varepsilon_{\mathrm{blob}}^{*}\right)^{1 / 3}\right]^{2} /\left[\delta^{3}\left(1-\varepsilon_{\mathrm{blob}}^{*}\right)\right]} \\
& =(\lambda R)^{2}\left(1-\varepsilon_{\mathrm{blob}}^{*}\right)^{-1 / 3}\left(\delta^{3}\right)^{-1}
\end{aligned}
$$

By eq 1 and 3 we rearrange the coordinate given by eq 19 as follows

$$
\left(\rho_{\mathrm{s}}-\rho_{1}\right) / \mu \cdot R^{2}\left(1-\varepsilon_{\mathrm{coil}}\right)\left(1-\varepsilon_{\mathrm{blob}}^{*}\right)^{-1 / 3} \cdot \frac{1}{s}
$$

Then we utilize the following equations for the molecular or blob weights $M$ and coil or blob parameters $^{1}$

$$
M=m R^{3}\left(1-\varepsilon_{\text {coil }}\right)
$$

where

$$
\begin{aligned}
& m=4 / 3 \cdot \pi \cdot \rho_{\mathrm{s}} / 1.66 \times 10^{-27} \\
& R=\left[\left(\frac{\text { const }_{3}}{\text { const }_{1} \text { const }_{4}{ }^{n}}\right)^{1 / n} \cdot m / M\right]^{-n /(3 n-1)} \\
& 1-\varepsilon_{\text {coil }}= \\
& {\left[\left(-\frac{\text { const }_{3}}{\text { const }_{1} \text { const }_{4}{ }^{n}}\right)^{3} \cdot m / M\right]^{1 /(3 n-1)}}
\end{aligned}
$$

to get

$$
\left(\rho_{\mathrm{s}}-\rho_{1}\right) / \mu \cdot m^{-2 / 3} M_{\mathrm{h}}^{1 /[3(3 n-1)]} M^{(2 n-1) /(3 n-1)} \cdot \frac{1}{s}
$$

where

$$
M_{\mathrm{h}}=m R_{\mathrm{h}}{ }^{3}
$$

is the blob weight at the uniformity concentration and corresponds to $1-\varepsilon_{\text {blob }}^{*}$ as calculated by eq 23 .

The second general conrdinate given by the formula 17 will not be changed. Rearranging in the same way we get

$$
\rho_{\mathrm{s}}^{-1} M_{\mathrm{h}}^{-1 /(3 n-1)} M^{1 /(3 n-1)} \cdot c
$$

where

$$
c=\rho_{\mathrm{s}}(1-\varepsilon)
$$

The coordinates described by the formulae 24 and 26 are the generalized ones to correlate the concentration and molecular weight dependences of sedimentation coefficient onto a single curve. The theoretical curve is generated by eq 18 in the coordinate system $(\lambda R)^{2} /(1-\varepsilon)-(1-\varepsilon)$ being the special case for hard spheres $\left(1-\varepsilon_{\mathrm{blob}}^{*}=1,1-\varepsilon_{\text {coil }}=1\right)$ of the coordinates given by the formulae 19 and 17 , respectively.

The general coordinates are applicable in both dilute and semidilute regimes. In the semidilute regime, however, $M$ does not mean the molecular weight but the blob weight and is to be calculated by the following equation obtained from eq $3,10,23$, and 27

$$
M=m \rho_{s}^{3 n-1}\left(\text { const }_{3} / \text { const }_{1}\right)^{3} / \text { const }_{4} \cdot c^{1-3 n}
$$

for

$$
c>\rho_{\mathrm{s}}\left[\left(\text { const }_{3} / \text { const }_{1}\right)^{3} / \text { const }_{4} \cdot m / M\right]^{1 /(3 n-1)}
$$

$M$ in eq 29 being the molecular weight.

Knowing the experimental values of $\rho_{\mathrm{s}}, \rho_{\mathrm{l}}$, $\mu$, const ${ }_{1}, n$, and hence $m$ by eq 21 and $M_{\mathrm{h}}$ by eq 15 and 25 , we are able to calculate the 


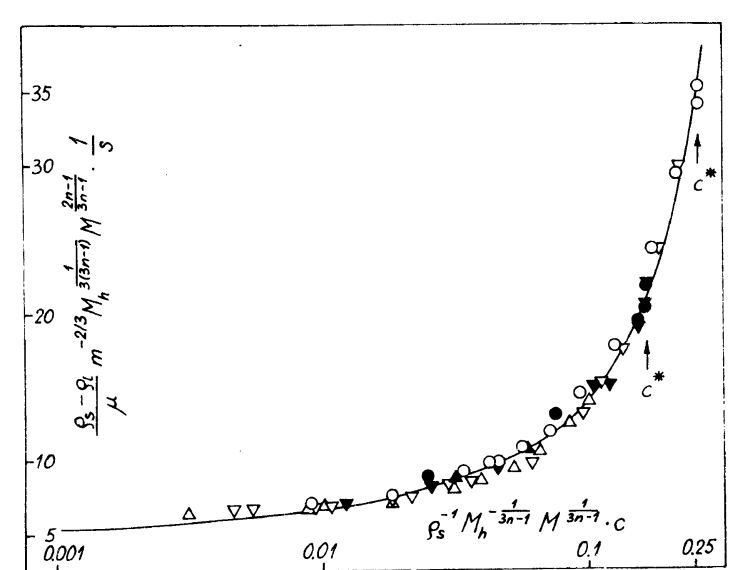

Figure 2. The generalized sedimentation data of PAMS of the following molecular weights: $M=2.34 \times 10^{5}(\triangle$, in cyclohexane; $\boldsymbol{\Delta}$, in toluene); $M=1 \times 10^{6}(\nabla$, in cyclohexane; $\nabla$, in toluene); $M=6.5 \times 10^{6}(\mathrm{O}$, in cyclohexane; in toluene). $c^{*}$ indicates the semidilute region.

generalized coordinates for given values of $s$, $c$, and $M$.

Such procedure was performed with the sedimentation data measured by Mijnlieff and Jaspers ${ }^{1,5}$ for poly $(\alpha$-methyl styrene) (PAMS) of three different molecular weights in cyclohexane and toluene. The systems analyzed greatly differ because cyclohexane is a poor solvent for PAMS and measurements were performed at the theta temperature, whereas toluene is a good solvent for PAMS and this system was investigated far from theta conditions. In spite of such a difference, the points in Figure 2 are quite close to one another and to the theoretical line.

The points marked by $c^{*}$ are the representation of the semidilute regimes. There are given two extreme points resulting from experimental errors, appearing in. ${ }^{5}$

\section{CONCLUDING REMARKS}

A hydrodynamic representation of the coils and blobs of a polymer dissolved, as consisted of hard spheres and pure solvent, was examined both theoretically and experimentally. A uniform approach is thus possible to study sedimentation of polymer solutions.

\section{REFERENCES}

1. L. Gmachowski, Polym. J., 18, 783 (1986).

2. L. Gmachowski, Polym. J., 18, 791 (1986).

3. B. Nyström, O. Waernes, and J. Roots, J. Polym. Sci., Polym. Lett., 27, 271 (1989).

4. L. Gmachowski, Polym. J., 20, 1073 (1988).

5. P. F. Mijnlieff and W. J. M. Jaspers, Trans. Faraday Soc., 67, 1837 (1971). 